\title{
Relationship of resting prolactin and testosterone in males during intensive training
}

\author{
A.C. Hackney, PhD, R.L. Sharp, PhD, W.S. Runyan, DSc and R.J. Ness, MA \\ Exercise Biochemistry Laboratory, Iowa State University, Ames, Iowa 50011 USA
}

Recently, findings of lowered serum testosterone levels and other hypogonadal symptoms have been reported in endurance trained males ${ }^{1,2}$. The mechanisms of these changes have been proposed to be similar to those put forth for females experiencing athletic amenorrhoea, a disruption of the hypothalamic-pituitary-gonadal axis. In preliminary findings, we have found a relationship between changes in resting serum prolactin and testosterone levels in males undergoing exercise training.

Nine healthy, athletic males were placed on an eight week intensive training programme ( 5 day/week, $\geqslant 90$ minutes/day). The training involved cycle ergometry, and contained a combination of continuous $(\sim 70$ per cent maximal aerobic power) and interval ( $\sim 80-150$ per cent maximal aerobic power) based training. Every two weeks the maximal aerobic power of the subjects was re-evaluated and new training loads prescribed. This training protocol was selected to mimic what competitive athletes typically perform. Resting (10 hour post-prandial) blood samples were collected every Monday morning, after two days rest, at two week intervals throughout the study. Table 1 shows the hormonal changes (mean \pm SEM) observed. Significant changes $(P \leqslant 0.05$; ANOVA) from pre-training levels are denoted with an asterisk.

The four to six week testosterone levels were significantly reduced while prolactin levels were significantly elevated during this time. Correlation analysis (Pearson-product) of the changes observed in the two hormones resulted in an $r=-0.934(P<0.01)$. These

Address for correspondence: A.C. Hackney, Exercise Physiology Laboratory, CB \# 8700 - Fetzer, University of North Carolina, Chapel Hill, North Carolina, 27599 USA
Table 1. Resting hormonal changes over the eight weeks of training

\begin{tabular}{lrrccr}
\hline & \multicolumn{5}{c}{ WEEKS } \\
& PRE & 2 & 4 & 6 & 8 \\
\hline $\begin{array}{c}\text { Testosterone } \\
\text { (ng/dL) }\end{array}$ & 800 & 664 & $534^{*}$ & $585^{*}$ & 794 \\
& \pm 122 & \pm 69 & \pm 92 & \pm 45 & \pm 112 \\
$\begin{array}{c}\text { Prolactin } \\
(\mathrm{ng} / \mathrm{mL})\end{array}$ & & & & & \\
& 7.7 & 13.6 & $15.9^{*}$ & $17.6^{*}$ & 10.9 \\
& \pm 2.0 & \pm 3.2 & \pm 2.5 & \pm 2.9 & \pm 2.5 \\
\hline
\end{tabular}

prolactin changes are at odds with some reports of suppressed prolactin levels in athletic males found to have lowered testosterone levels ${ }^{2}$, but agree with changes reported for women undergoing endurance training and experiencing menstrual abnormalities ${ }^{3}$.

Our findings suggest that suppressed testosterone during short-term training periods may be associated with elevations in prolactin, but if a true cause and effect relationship exists it cannot be determined. Because of our limited sample size, these present findings have to be viewed tentatively. However, currently we are attempting to confirm the relationship found in this study.

\section{References}

1 Hackney, A.C., Sinning, W.E. and Bruot, B.C. Reproductive hormonal profiles of endurance trained and untrained males Med Sci Sports Exerc 1988, 20, 60-65

2 Wheeler, G.D., Wall, S., Belcastro, A.N. and Cumming, D.C. Reduced serum testosterone and prolactin levels in male distance runners JAMA 1984, 252, 514-516

3 Boyen, T.W., Pamenter, R.W., Grosso, D., Standforth, P., Rotkis, T. and Wilmore, J.H. Prolactin responses, menstrual cycle, and body composition of women runners J Clin Endocrinol Metab 1982, 54, 711-714 